\title{
5G New Radio in SATCOM: An Overview of Physical and Medium Access Layer Issues
}

\author{
Harri Saarnisaari ${ }^{1}$, Senior Member, IEEE, Carlos Morais de Lima, Member, IEEE \\ University of Oulu, Oulu, Finland \\ Tel: (+358) 405727803, e-mail: harri.saarnisaari@oulu.fi
}

\begin{abstract}
The 3GPP standardization body is actively investigating the requirements and specifying the radio access interface of $5 \mathrm{G}$ new radio (NR) systems to support satellite communications. However, the 5G NR was originally designed as a terrestrial system, therefore, there are a few open questions and technological challenges that need to be assessed so as to properly support such non-terrestrial communication systems. In that regard, several related problems and potential solutions have been proposed and evaluated not only within 3GPP working groups, but also by the scientific community including academia and industry. In this contribution, we first overview the recent developments in the random access and physical layer procedures to support satellite communication in $5 \mathrm{G}$ networks, identify their limitations and then propose viable solutions. Note that such solutions do not necessarily require to change the standard, but eventually to reparametrize the 5G NR signal based on vendors knowledge of specific deployment scenarios. For instance, we observed that the proper use of reference symbols is required to cope with nonlinear effects due to satellite amplifiers. Our results also show that high reference symbol density is required, while still just fairly low modulation orders can be used.
\end{abstract}

Keywords: initial access, random access, mobile networks, satellite systems.

\section{INTRODUCTION}

Lately, the integration of satellite communications and wireless terrestrial networks is under active discussion in the context of $5 \mathrm{G}$ NR systems. In fact, the benefits of incorporating spaceborne vehicles into such $5 \mathrm{G}$ networks, the undeniable satellite effectiveness to provide ubiquitous coverage, as well as the needed interoperability between distinct radio access interfaces has been studied in 3GPP since release 14 [1]. In that regard, the 3GPP introduced the concept of non-terrestrial networks (NTN) which use airborne or spaceborne vehicles for transmission and investigated implementation features and possible network architecture options [2].

Hitherto, (non) terrestrial systems have been designed independently and co-operation has been possible only via gateway solutions such that, for example, satellite systems are already supported in $4 \mathrm{G}$ systems, tough with limited interoperability. However, since 2018 the satellite element has been integrated into the 3GPP 5G new radio (NR) standardization work [2] which enables a seamless interoperability between both terrestrial and nonterrestrial networks. The identified use cases are backhaul usage and direct user access. Integration should be done in all levels. Currently, key technologies introduced by the 5G NR are still undergoing integration, for instance, high level softwarization of network functions, related management and orchestration as well as distributed network functions, slicing, multi access edge computing among others.

One integration goal is to allow use $5 \mathrm{G} \mathrm{NR}$ in satellite systems since that offers several benefits like reduced costs. This requires that suitability of $5 \mathrm{G}$ NR to satellite systems has to be analysed also in the signal level. Last year in ICTON, the authors summarized progress so far [3] and in this paper summarization is continued. The paper briefly reminds earlier results and then concentrates on new results such that the paper forms a solid information source.

The signal level means physical (PHY) and medium access (MAC) layers. The main sources of challenges to $5 \mathrm{G} \mathrm{NR}$ are the long propagation delay of satellites and large Doppler frequency shift on some satellite systems. Low Earth orbit (LEO) satellites are up from $600 \mathrm{~km}$ altitude and others still higher whereas in $5 \mathrm{G}$ NR the largest cell size is $300 \mathrm{~km}$. LEO satellites move $7200 \mathrm{~km} / \mathrm{s}$ whereas high speed trains a few hundred $\mathrm{km} / \mathrm{h}$ and result in over $700 \mathrm{kHz}$ carrier frequency offset (CFO) at $30 \mathrm{GHz}$ carrier [2].

This paper first discusses MAC layer issues where the propagation delay in the main source of challenges. In particular, initial random access process in considered. Secondly the paper considers the PHY layer issues. First, initial downlink synchronization, where large CFO is the main source of problems, is considered. Thereafter, problems raising from (satellite) high power amplifier (HPA) are considered. The concern is that satellites like to operate at a small back-off region and 5G NR signal with its large peak-to-average power ratio (PAPR) is not well suited for that. Some solutions to this are discussed. This overview is based on the work done in the SaT5G project [http://sat5g-project.eu/].

\footnotetext{
${ }^{1}$ The work has been financially supported by EU Horizon 2020 project SaT5G under grant agreement No 761413, and Academy of Finland 6Genesis Flagship (grant 318927).
} 
There is one obvious solution for these problems except high PAPR. It is the use of device's location and satellite trajectory information and it is included into 3GPP toolkit of solutions in the NTN integration. It allows compensating propagation delays and CFOs. However, this is not necessarily available for all devices such that alternative solutions, discussed herein and within 3GPP working groups, are needed.

The discussed topics are not the only ones. Furthermore, they are not necessarily affecting the 5G NR standard but just for vendors and how 5G NR is actually used, i.e., parameters selected. For example, the standard does not describe scheduler algorithms, it just describes preconditions. And still it would be advantageous if vendors would design satellite system friendly schedulers, as also mentioned in the MAC layer analysis. This would increase the throughput since satellite propagation delays could be taken into account in an optimized way.

\section{RANDOM ACCESS}

Succinctly, the random access (RA) procedure in 5G NR systems is employed to: $i$ ) synchronize with new serving cells when performing handover; $i$ ) resynchronize with the current serving cell after long periods of inactivity; and iii) request uplink scheduling opportunities during initial access. Before the initial RA procedure, the NTN terminal (or user equipment, UE) has to read system information from the synchronization signal (SS) block (and elsewhere if needed). Therein, the serving base station (gNB) schedules NTN terminals to transmit the preamble information over specific time/frequency resources. It is interesting to note that also satellites could have gNB functionalities, if they are regenerative.

If it wants access, the NTN terminal sends a RA preamble in a physical random access channel (PRACH). Upon reception of it, gNB calculates a timing advance (TA) value for that NTN terminal and sends it in a random access response (RAR) signal to the terminal along with other information. The process includes timers, but these are not a problem since 5G NR value range for them is so large, if just set properly [8]. Another thing is that the PRACH signal must be accompanied by a guard interval (GI) such it does not overlap with other signals. In what follows, GI and TA issues are discussed, respectively.

\subsection{PRACH Guard Interval}

The 5G NR standards do not specify any particular guard interval; hence, it can be easily adapted to match different cell sizes, even the large coverage areas commonly encountered in satellite communication systems. Indeed, it is a task of the scheduler to set it and not allocate other signals for that period [4].

Usually we would set the GI value based on the round-trip time (RTT) between the satellite and the longest link relative to the beam coverage area also known as the cell footprint. Note that satellite systems undergoes much larger RTTs than those experienced in terrestrial systems starting from $4 \mathrm{~ms}$ LEO satellite systems at 600 $\mathrm{km}$ altitude and ending to few hundreds of $\mathrm{ms}$ in geostationary orbit (GEO) satellites. This means that the scheduler has to reserve significant amount of resources just for GI, which is the principal problem concerning this subsection.

In this context, the so-called differential distance (DD) concept illustrated in Figure 1 has been introduced to reduce the required GI in non-terrestrial communication systems. By using the DD approach, an NTN terminal first subtracts the shortest distance from the satellite to the footprint, and then uses the remaining delay as the one that needs to be covered by GI as the difference between the maximum and minimum delays. The minimum distance could be per beam and it could be sent dynamically since the beam shape may change; or alternatively the minimum distance would be a new system information that needs to be defined in the 5G NR standard. In this configuration, the footprints may vary from a few hundred $\mathrm{km}$ up to several thousand $\mathrm{km} \mathrm{[2].}$

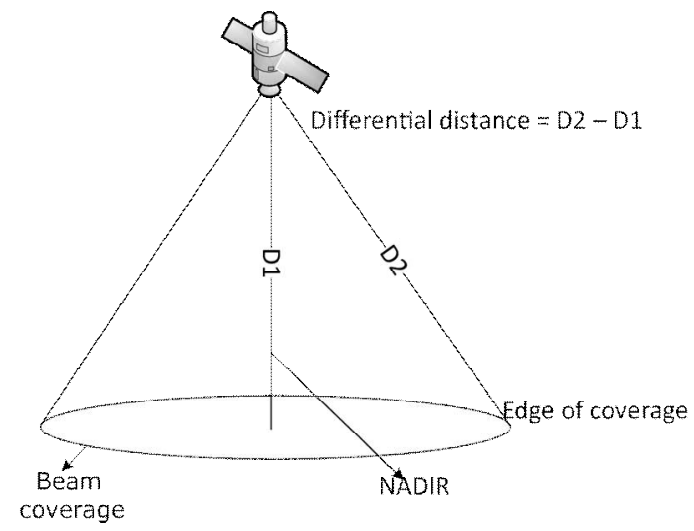

Figure 1. Illustration of the differential delay concept. 
By employing the DD concept, it is also possible to reduce the PRACH utilization intervals and thus improve its overall capacity. According to the current implementation in the specifications, it is possible to vary the slots periodicity of the RACH from $10 \mathrm{~ms}$ to $160 \mathrm{~ms}$ (the interested reader needs to check Section 16.2 in [4].) This is a viable option since handovers do not necessarily occur very frequently in satellite systems, especially in backhaul configuration where NTN terminals (as part of gNB) tend to have almost continuous backhaul needs.

\subsection{Timing Advance Value}

The RAR message has 12 bits reserved for TA, that matches $300 \mathrm{~km}$ at $15 \mathrm{kHz}$ sub carrier spacing (SCS), 150 $\mathrm{km}$ at $30 \mathrm{kHz}$ SCS and so on. This is not adequate for satellite systems. One method to overcome this is simply add extra bits to the RAR message or by determining an alternative mean to transmit the TA value bits. In addition, TA value calculation in $\mathrm{gNBs}$ has to be redefined.

The second overcoming method employs the DD approach which in this case is called the differential delay concept. According to this approach, extra bits are only needed in large footprints, whereas no extra bits for TA value are not needed if the satellite footprint is small. This approach becomes more beneficial in high altitude satellite systems like GEO and medium Earth orbit (MEO) satellite systems because it is possible to considerably reduce the associated delays. More detailed analysis of these random access issues can be found in [8].

\section{PHYSICAL LAYER}

\subsection{Initial Downlink Synchronization}

During initial downlink synchronization an NTN terminal tries to detect existing base stations, get initial timing and read information about access parameters. These are in the SS block that contains the primary and secondary synchronization signals (PSS and SSS) as well as the system information data that is heavily coded using a polar code [4].

Usual 5G NR receivers fail to detect the PSS signal if CFO is too large. Therefore, a large CFO aware receiver is needed [5]. This is a vendor problem since the standard just defines the environment. If it will not be decided that position-and-trajectory information is mandatory in NTN devices, the standard has to specify a larger CFO range where NTN receivers have to operate, and vendors have to react correspondingly.

A large CFO aware receiver basically has several branches each matched for different candidate CFO [3]. The number of branches depends on CFO uncertainty range and duration of the PSS signal as discussed in [5]. It was shown that the PSS signal can be detected based on 5G NR targets, i.e., at $-6 \mathrm{~dB} / \mathrm{RE}$ level (RE stands for resource element or active subcarrier). After detecting the PSS signal and correcting carrier frequency by the obtained initial CFO estimate, detection of the SSS signal as well demodulating and encoding data in the SS block can be done using a normal receiver [5].

\subsection{High PAPR Problem}

Low HPA back-off is essential in satellite systems since that would maximize (average) signal level and, consequently, signal quality, and in satellite systems signal power is typically rather low anyway. 5G NR signal is an orthogonal frequency division multiplexing (OFDM) signal and therefore known of its high PAPR value, above $10 \mathrm{~dB}$. If such a signal is passed through a high power amplifier with low back-off, it would be clipped. This clipping distorts the signal and also causes undesired out-of-band emissions.

As a solution, one should apply PAPR reduction techniques that also limit out-of-band emission. There is a rich literature of these, but a majority of studies is concentrating on moderate level PAPR levels (around $6 \mathrm{~dB}$ ) and do not consider very low levels targeted herein. At moderate PAPR levels, signal distortion effects to bit error rate (BER) or block error rate (BLER) are not so obvious. Furthermore, the technique should be receiver agnostic since $5 \mathrm{G}$ NR does not support other solutions (at least at the moment).

An iterative clipping and filter methods is such a method. It goes down to a desired PAPR level and it is relatively simple to implement. It is located at the baseband before the signal is transformed into the carrier frequency as illustrated in Figure 2. Usually it is applied with rather high level oversampling, but it was shown in [9] that natural oversampling of 5G NR signal is sufficient (zeros at the band edges). The desired PAPR level in [9] was $2 \mathrm{~dB}$ that was also the back-of level of used solid state power amplifier model. It was shown that the SS block data can be received without distortion if compared to receiver without PAPR reduction and nonlinear HPA. However, SS block's QPSK data is heavily coded, by a rate 1/5 code. In addition, reference symbols are dense at every $4^{\text {th }}$ subcarrier in each OFDM symbol of the SS block.

The data part rarely uses such a strong coding but targets lower rates as well as for higher order modulations in order to achieve higher data rates. It was shown in [9] that QPSK modulation and $1 / 2$ rate coding are possible with $2 \mathrm{~dB}$ PAPR level (and maybe a bit lower) if the density of the reference symbols is sufficient [9]. It was shown that if subcarrier density is relaxed, the losses are larger, i.e., sensitivity degreased. Furthermore, it was concluded that work is still needed to find sufficient reference symbols density for each modulation-and-coding scheme, to see if higher order modulations are possible and what is the ultimate PAPR limit. These selections are 
not affecting the 5G NR standard since the standard is flexible while reference symbols are set, and it readily supports various modulation-and-coding combinations. The purpose is to help operators to select optimal parameter sets for satellite use, satellite operators to set the back-off level vs desired channel quality and data rate, as well as vendors to design proper PAPR and out-of-band emission schemes.

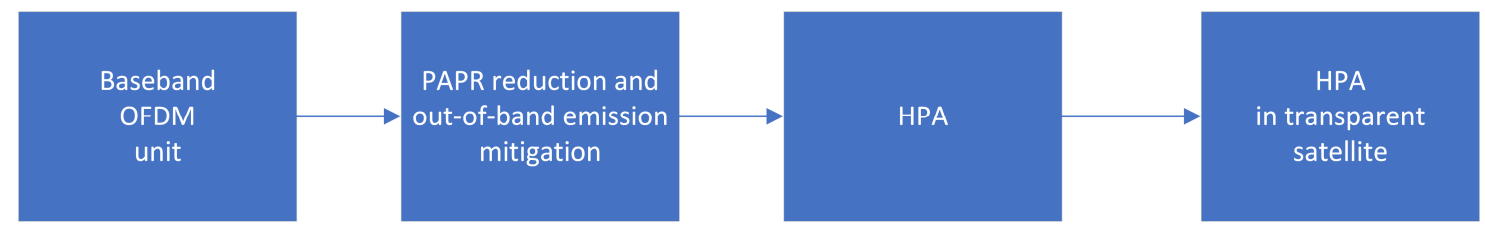

Figure 2. An OFDM transmitter block diagram with PAPR reduction with a transparent satellite.

\section{CONCLUSIONS}

This paper overviews medium access and physical layer challenges when 5G NR signal is applied for satellite links. It shows the origin of challenges and discusses possible solutions. The main sources of challenges are long propagation delay and large Doppler frequency shift, the latter especially in LEO systems. Use of device location and satellite trajectory information would overcome many challenges, but that is not mandatory requirement and other solutions discussed in the paper are needed.

It was shown that differential delay concept is beneficial in reducing the required excessive TA value in satellite systems as well as to reduce the GI of the random access signal. Otherwise, resources are wasted for GI and more bits are needed for TA in the RAR message. Usual receiver is not sufficient if CFO is large, but a large CFO aware receiver has to be used. The extra processing concerns initial SS signal detection and initial CFO estimation. Thereafter, usual receiver is sufficient. Finally, it was shown that using the iterative clipping and filtering method to reduce PAPR and out-of-band emissions, it is possible to achieve $2 \mathrm{~dB}$ PAPR level with QPSK modulation and $1 / 2$ rate coding assuming that reference symbols density is sufficient. If it is not, more SNR is required. Open problems were also discussed.

\section{ACKNOWLEDGEMENTS}

This paper is based on results obtained in the Horizon 2020 SaT5G project under grant agreement No 761413 that aims to study integration of satellite and $5 \mathrm{G}$ terrestrial systems in a wide sense, from many angles. This research has also been supported by Academy of Finland 6Genesis Flagship (grant 318927).

\section{REFERENCES}

[1] 3rd Generation Partnership Project; Technical Specification Group Services and System Aspects, "Service requirements for the 5G system; Stage 1," 2019, 3GPP TS 22.261 V17.1.0 (2019-12).

[2] 3rd Generation Partnership Project; Technical Specification Group Radio Access Network, Study on new radio (NR) to support non terrestrial networks (release 15), 2018, 3GPP TR 38.811 V15.0.0 (2018-06).

[3] H. Saarnisaari, C.M. de Lima, "5G NR over satellite links: Evaluation of synchronization and random access processes“, 1st Workshop on Integration of Optical and Satellite Communication Systems into 5 G Edge Networks in 21th International Conference of Transparent Optical Network (ICTON).

[4] E. Dahlman, S. Parkval, and J. Sköld, 5G NR: The Next Generation Wireless Access Technology, Academic Press, 2018.

[5] H. Saarnisaari, J-M. Houssin, T. Deleu: 5G New Radio Over Satellite Links: Synchronization Block Processing, EuCNC, 2019.

[6] 3GPP TR 38.300 V15.0.0, "Technical Specification Group Radio Access Network; NR; NR and NRRAN Overall Description; Stage 2 (Release 15),” Technical Report, 2018.

[7] 3GPP TR 38.213 V15.0.0, "Technical Specification Group Radio Access Network; NR; Physical layer procedures for control (Release 15)," Technical Report, 2018

[8] H. Saarnisaari, A. Laiyemo, C. M. de Lima: Random Access Process Analysis of 5G New Radio Based Satellite Links, Satellite and Non-Terrestrial Networks for 5G Workshop in IEEE 5G World Forum, 2019.

[9] H. Saarnisaari, C. Lima C., Integrating 5G NR and Satellites Systems: Main features, Needed Changes and Performance Results, submitted to IJSCN Special Issue on "Satellite Networks Integration with 5G", March 2020. 